\title{
Risk Factors and Sleep Intervention Considerations in Esports: A Review and Practical Guide
}

\author{
Daniel Bonnar, MPsych¹, Sangha Lee, $\mathrm{MA}^{2}$, Michael Gradisar, $\mathrm{PhD}^{1}$, Sooyeon Suh, $\mathrm{PhD}^{2}$ \\ ${ }^{1}$ College of Education, Psychology and Social Work, Flinders University, Adelaide, South Australia, Australia \\ 'Department of Psychology, Sungshin Women's University, Seoul, Korea
}

Received: November 30, 2019 Accepted: December 16, 2019

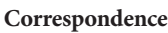

Esports is a booming global industry and has been officially included in the lead-up to the 2020 Olympics in Tokyo. Given that esports is a cognitive based activity, and sleep is well known to be critical for optimal cognitive functioning, our research group recently proposed that sleep might be an important determinant of esports performance. The focus of the current review was to expand our limited understanding regarding the role of sleep in esports by exploring risk factors for suboptimal sleep and developing an associated intervention framework. More specifically, we aimed to 1) examine how gaming culture and game genre might negatively influence sleep behaviour, 2) describe a conceptual model to explain how sub-optimal sleep occurs in esports, and 3) outline sleep intervention considerations that specifically meet the needs of esports athletes. We conclude that gaming culture and game genre could both impact the sleep behaviour of esports athletes, via cognitive and behavioural mechanisms. Furthermore, adapting Spielman's three-factor model to esports may provide a useful and easy to understand conceptualisation for sub-optimal sleep in esports. Lastly, sleep interventions for traditional athletes can be suitably modified for esports but must be comprehensive and extend from a theoretically grounded conceptual model.

Sleep Med Res 2019;10(2):59-66

Key Words Sleep, Esports, Risk factors, Intervention, Performance.

\section{INTRODUCTION}

Esports, a form of organised video game competition [1], has developed from relative obscurity into a billion dollar global industry. In 2018 there were an estimated 380 million spectators worldwide [2], while in 2019 the Fortnite World Cup champion won more prize money than Novak Djokovic and Simona Halep received for winning Wimbledon [3,4]. More recently, it has been announced that esports will officially be included in the lead up to the 2020 Olympics [5]. Taken together, these developments clearly demonstrate the esports industry's accelerating growth, popularity and increasing professionalisation [2]. Yet, despite the transformation esports is currently undertaking, there is limited performance-based research available to support the needs of the competitors themselves. Thus, for esports athletes to reach their peak potential, research is required to address this gap in the literature.

Esports is a cognitive based activity [6] and sleep is critical for optimal cognitive functioning (e.g., processing speed, working memory, executive functioning) [7,8]. Hence, our research group has recently proposed that adequate sleep likely plays a key role in esports performance [9]. Importantly, despite the apparent need for sleep, esports athletes may be exposed to a range of risk factors for sub-optimal sleep similar to traditional athletes. In our previous paper [9], we outlined several universal risk factors (e.g., caffeine use, pre-competition anxiety, travel) experienced by all athletes regardless of the sport played. However, other potential risk factors for 
sub-optimal sleep inherent to esports also warrant attention, namely, gaming culture and game genre. Neither of these factors has been examined in relation to sleep although both may have the capacity to be sleep disrupting. Additionally, as an increasing number of risk factors are identified, it will prove useful to have a conceptual model outlining how they contribute to the development and maintenance of sleep disturbances in esports.

Furthermore, given the unique conditions and experiences that characterise esports, there is a need to consider how sleep interventions should be designed and delivered to esports athletes. Over recent years there has been rapid development in sleep management strategies specifically designed to address the idiosyncratic needs of athletes in traditional sports, with a general push toward intervention 'packages' over isolated strategies [10]. Although many strategies used within traditional sports may be generally appropriate (e.g., sleep education, relaxation techniques) in esports as well, the specific content, aims, style, and mode of delivery, technology, resources, and evaluation tools used, should be devised based on the sleep needs of esports athletes. To the best of the authors' knowledge, no previous paper has attempted to explore this topic.

The focus of this review is to expand the available scientific knowledge on risk factors for poor sleep in esports and inform 'on the ground' practices regarding the sleep management of esports athletes. Our aims are to, 1) explore how gaming culture and game genre may negatively impact sleep, 2) describe a conceptual model that outlines the development and maintenance of sub-optimal sleep in esports, and 3) outline considerations for sleep interventions tailored specifically to the needs of esports athletes.

\section{RISK FACTORS FOR POOR SLEEP IN ESPORTS}

In the following section we focus on two potential risk factors for poor sleep unique to esports, namely, gaming culture, and game genre. More specifically, we draw from the broader sleep, sporting, and gaming literature to describe the potential mechanism by which these factors may impact sleep in esports.

\section{Gaming Culture}

Culture (defined as the shared attitudes, values, and customs of a particular group) [11] has been found to have a role in shaping beliefs, which in turn, influence behaviour, including sleep [12]. In their review on traditional sports, Burgess and Naughton [13] outlined how junior athletes who show potential are selected for talent development pathways to help propel them to elite levels. During this development phase, they are often afforded organisational support in the form of education while performance-enhancing behaviour, such as adopting adequate nutrition and obtaining sufficient sleep [14], are promoted. Es- sentially, from a young age, traditional athletes are immersed in a culture in which performance-enhancing behaviour, informed by a strong foundation of sports science research, is prioritised in order to to increase the likelihood of competitive success [13]. Hence, athletes may be more likely to engage in behaviour consistent with this type of culture and the evidence-based beliefs that develop in response. For example, an individual who has a belief that they need to keep a regular sleep-wake schedule to obtain adequate sleep may be less likely to stay up late watching Netflix and instead keep a consistent bedtime and wake-up time.

In comparison, the pathway to professional esports is far less institutionalised, and is predominantly unregulated and unstructured [15]. Taylor [15] notes that because junior leagues are uncommon, many esports athletes start out as amateur gamers (i.e., individual, casual players of video games), and spend their formative years playing online or possibly at LAN (i.e., local area network) events face-to-face against other amateur gamers. In other words, they exist in an environment that generally lacks the organisational oversight, support, and educational privileges received by traditional athletes. Furthermore, they often belong to or play with a particular group (sometimes known as a clan or guild), which can take on its own sub-culture and group norms. The collective consequence is that unhelpful and dysfunctional beliefs can develop from this sub-culture. For example, the gaming community often promotes 'grinding' (i.e., excessive practice, up to $13 \mathrm{hrs}$ p/day) over other health considerations such as nutrition, physical activity and sleep, presumably due to perceived performance benefits (e.g., skill mastery) [16]. Although the benefits of grinding have not been empirically tested, literature on the diminishing returns of sleep loss relating to cognitive performance [17] would suggest this strategy is unhelpful.

It should be noted that the esports athlete development landscape is making positive progress, albeit slowly. For example, academies and minor leagues are being implemented at a college/university and high school level. Moreover, although performance-based research is still in its infancy, this too is beginning to change. However, there is a still a long way to go before esports athlete development reaches a level of professionalisation similar to that seen in traditional sports.

Unhelpful and dysfunctional beliefs about sleep amongst gamers may also be reinforced by other segments of the wider gaming community such as gaming 'streamers' (i.e., an individual who broadcasts their gaming activity live to online viewers [18]). Richard Blevins (as known as 'Ninja'), one of the most popular streamers in the world who had a total of 478 million views while on Twitch (a popular streaming platform) streamed on average for $8.8 \mathrm{hrs}$ at a time and usually started at $2: 50 \mathrm{pm}$ (with a finish time of approx. 11:39 pm) [19]. Furthermore, in April 2019, a streamer named 'Edison Park' streamed content for approximately $17 \mathrm{hrs}$ p/day over 30 days for a total of $541 \mathrm{hrs}$ [20]. Social learning theory [21] would suggest that this type of 'play till you drop' behaviour modelled by popular and influen- 
tial streamers could serve to de-value the importance of sleep in the eyes of viewers, which includes future professional esports athletes.

Consequently, it is plausible that in the absence of intervention, unhelpful and dysfunctional beliefs about sleep shaped by gaming culture during the formative years in amateur gaming environments could be carried unmodified into professional esports. Hence, at elite levels when optimal performance is paramount for competitive success, some esports athletes may be pre-disposed toward unhelpful wake behaviours that compromise sleep and in turn their performance. Furthermore, if an esports athlete plays in a team with others who hold similar beliefs, this would also perpetuate poor sleep behaviour. In sum, gaming culture would appear to have the capacity to negatively influence sleep, although empirical research is required to determine the exact nature and extent of this influence.

\section{Game Genre}

Similar to traditional sports, there are several genres in esports. The most popular genres are real-time strategy (RTS), multiplayer online battle arena (MOBA), first person shooter (FPS) and sports games. RTS is a strategy game genre where the goal is to win a battle by competing with opponents, securing resources, and producing and operating units [22]. The most popular game of the RTS genre is Starcraft. FPS is a game genre where the player runs through a three-dimensional space and attacks the enemy by firing a weapon such as a gun [23]. The most popular games of the FPS genre are Counter Strike: Global Offensive (CS:GO) and Overwatch. MOBA developed from the RTS genre [24] and combines a match and a siege (game genre aimed at attacking a building of a partner). There are many different MOBA games, but League of Legends (LoL) and Defense of the Ancients 2 (DOTA2) are the most popular [25]. The sports genre in esports refer to games that simulate the sporting experience including sports such as soccer, baseball, golf, basketball, and so forth. FPS and MOBA genres are team esports, and RTS and sports genres are mainly individual esports [22].

The sleep of esports athletes may be affected by the type of genre in which an individual specialises. There is currently little empirical evidence to support that some genres have more influence on sleep compared to others. However, the idea of specific sport genres having differential effects on sleep has been examined in traditional elite athletes.

Nedelec et al. [26] proposed that sleep of elite athletes is affected by multiple sport-specific and societal factors. The research in traditional sports has been classified in two ways: one is by the team format (individual vs. group), and the other is by the characteristics of the sport. The literature focusing on team size reported that total sleep time of athletes from individual sports was 30 minutes less than those who engaged in team sports, even though they went to bed earlier and woke-up earlier [27]. This result suggests that individual sports athletes may take more naps during the daytime compared to those who engage in team sports to compensate for shorter night-time sleep durations. Similarly, it is possible that these results are reflected in esports, where esports athletes engaging in individual genres who do not have teammates and train individually at home (e.g., esports athletes playing FIFA online) can take a nap when they want to and have more freedom in deciding their sleep schedule.

When it comes to a type (or genre) of traditional sports classified by characteristics, sleep disorders are more common in some specific sports than others [28]. For example, sleep problems such as difficulty falling asleep, night time awakenings and daytime sleepiness are more prevalent in aesthetic sports (i.e., gymnastics, synchronised swimming, and figure skating), while athletes in high risk sports (i.e., sliding, aerial, and motor sports) report significantly less sleep issues than the others [29]. Obstructive sleep apnea seems to be much more prevalent among strength power athletes (e.g., rugby) than other athletes due to a high body mass index (BMI) score [30]. There are many studies that have reported on the correlation between game play time and obesity [31-33]. Ballard et al. [33] reported that the duration of gaming was positively associated with BMI in a study including 116 male participants. In a study comprising 10984 participants, Dunton et al. [34] reported a positive association between gaming and BMI. Considering that esports players spend more time playing games than regular gamers, it is possible that some esports players have a high BMI.

Suppiah et al. [35] also reported differences in sleep patterns between high-intensity athletes and low-intensity athletes. Highintensity athletes displayed significantly greater amounts of slowwave sleep, less light sleep, and better sleep continuity. It is difficult to ascertain which genre in esports are more intensive than others because esports does not require a lot of physical movement, and esports athletes are sedentary even when they are in the middle of the most challenging competitions. Nevertheless, similar to various shooting disciplines, the physical skills required for esports rely on fine, rather than gross, motor movements. These movements are measured as actions per minute (APM), which is the measure of how many clicks and key presses a player can perform in 60 seconds. These require manual dexterity to perform and are significantly and positively correlated with performance in esports [16]. Some esports genres may require high levels of APM compared to others. For example, in Starcraft 2 (RTS), in which fine control with fast hand speed is critical to win, elite esports athletes showed APMs up to 500 while other MOBA genres such as League of Legend (MOBA) does not require APMs that are as high [36]. While APM is different from the intensity of traditional sports, future research on the amount and density of fine motor skills used in specific esports genres and the differential effects on sleep should be measured and analysed.

In summary, sleep interventions for esports athletes can vary based on genre, depending on team size and game characteris- 
tics. Particularly, in the case of team esports, it may be necessary to design sleep interventions that take into account the whole team, overall schedule, characteristics of the individual teammates, and the sleep environment.

\section{CONSIDERATIONS FOR SLEEP INTERVENTIONS IN ESPORTS}

In the following section we initially adapt Spielman's threefactor model (3-P model) to conceptualise how sub-optimal sleep occur in esports, and then based on this model, we outline considerations for sleep interventions that address the specific sleep needs of esports athletes.

\section{Using the Cognitive Behaviour Therapy for Insomnia Framework for Esports Athletes}

Cognitive behaviour therapy for insomnia (CBT-I) is a nonpharmacological treatment for insomnia patients and is recommended as the first line of treatment for insomnia [37]. CBT-I is usually delivered as a multi-component treatment package consisting of behavioural and cognitive components delivered over 4 to 8 weekly sessions. The components consist of sleep hygiene, stimulus control, sleep restriction therapy, cognitive therapy, and relaxation training [38]. For a detailed review of each treatment component [38]. While not all esports athletes will have insomnia symptoms (e.g., difficulty falling asleep or difficulty staying asleep despite given ample opportunity), using the CBT-I framework to approach sleep disturbance in these players may be helpful in both preventing and treating future occurrences of sleep disturbance.

CBT-I is based on the 3-P model by Spielman et al. [39], and proposes that three factors are involved in the development and maintenance of insomnia: predisposing factors, precipitating factors, and perpetuating factors. Predisposing factors are vulnerabilities that an individual possesses which increase the likelihood of developing insomnia, such as genetic predisposition to sleep disturbance, being at high risk for psychopathology, or cir- cadian misalignment (i.e., being an extreme morning or evening type). For esports athletes, this may be strong tendencies toward eveningness. Additionally, most esports athletes are younger, which may developmentally predispose them to have strong eveningness tendencies during the height of their career $[40,41]$. Precipitating factors are usually stressful events that typically result in acute sleep disturbance, such as an exam, loss of a family member, or a break-up [42]. Instead of exams, esports athletes may experience sleep disturbance before a competition, similar to traditional elite athletes [43]. Additionally, unlike traditional athletes, esports training (as known as "scrims") and matches may take place late in the evening hours, which may delay sleep timing as is seen with young people's part-time work [44]. Frequent travel for matches and adjusting to several time zones in short periods of time may also be a precipitating factor for this population. While short-term sleep disturbance may be a natural stress response, some individuals may begin to engage in behavioural changes to battle the sleep disturbance that end up maintaining sleep problems. Perpetuating facts are maladaptive thoughts and behaviors that are usually initiated in an attempt to battle sleep disturbance, but end up contributing to the maintenance of sleep disturbance. For example, esports athletes may consume excessive amounts of caffeine to maximise game performance and also battle sleepiness. Additionally, late night training and matches may leave little time for esports athletes to wind down before bed after periods of high stress and excitability. In addition to changes in sleep-related behaviours, sleep-related cognitions can also perpetuate sleep disturbance. An esports athlete who is on a losing streak may believe that sacrificing sleep to train longer hours may curtail sleep duration, creating a vicious cycle of sleep deprivation, and poor game performance. The 3-P model suggests that sleep disturbance is a combination of all three factors, and designing sleep interventions for esports athletes will also need to take a comprehensive approach and consider all factors.

\section{Current Guidelines for Sleep in Traditional Athletes}

Previous studies have investigated sleep interventions in tra-

Table 1. Potential 3-P factors for esports players

\begin{tabular}{ll}
\hline Predisposing factor & - Strong eveningness tendencies \\
& - Gaming culture \\
Precipitating factor & - Late game and training sessions \\
& - Peak training workloads \\
& - Frequent travel (e.g., jet lag, hotel bed, noise) \\
& - Participating in team esports \\
& - Competition \\
& - Drinking excessive amounts of caffeine to enhance performance and battle sleepiness \\
& - Lack of wind-down time \\
& - Sleep-interfering activities (e.g., using smart phones in bed) \\
Perpetuating factor & - Irregular sleep-wake schedule \\
& - Dysfunctional beliefs about sleep (e.g., "I need to stay up longer to perform better")
\end{tabular}


ditional athletes to enhance physical and cognitive abilities. A review by Bonnar et al. [10] categorised sleep interventions for traditional athletes into three subtypes: 1) sleep extension and napping interventions, 2) interventions to promote sleep hygiene, and 3) post-exercise recovery strategies.

Sleep extension interventions target chronic sleep deprivation in this population, which recommends spending 9-10 hours in bed per day, including naps. Two studies that have investigated sleep extension and napping interventions both found an increase in total sleep duration and a corresponding increase in sports performance $[45,46]$. Similarly, napping interventions ask athletes to take daytime naps that are placed within the optimal circadian window (e.g., midday naps for swimmers) [10].

Interventions that promote sleep hygiene are typically behaviours that promote sleep, such as tending to the temperature and light of the bedroom environment, or avoiding alcohol or strenuous exercise before sleep [47]. While sleep hygiene is usually not recommended as a standalone treatment, studies have shown that a sleep hygiene intervention can be beneficial for increasing sleep duration for athletes [47].

Studies that have investigated post-exercise recovery interventions involve introducing procedures such as whole-body cryostimulation [48] and red light irradiation [49] with the goal of indirectly improving nocturnal sleep through these procedures. While the results of these studies look promising in improving sleep, more studies are needed to understand the relationship between these interventions, sleep and performance [10].

The literature on sleep interventions for traditional athletes is far from comprehensive and further research is needed to incorporate specific characteristics of the athlete lifestyle. Specifically, studies investigating the characteristics of esports athletes and their idiosyncratic sleep needs are necessary in tailoring sleep interventions for this population. In their study, Baltezarević and Baltezarević [25] noted that the typical age of esports athletes is between 17-25 years, an age that is particularly prone to strong evening tendencies [41]. Professional team members from countries such as South Korea and the US typically live together to improve training efficiency, which may make it challenging to have a sleep environment that is sleep-conducive. Thus, coaches and managers of esports teams who usually make important decisions about the athletes' lifestyle and training schedule should be incorporated into planning of sleep interventions for these athletes. This participation would improve their knowledge of sleep and also monitoring of their own sleep, which is important in promoting a top-down healthy sleep culture [50,51].

\section{Treatment Guidelines for Sleep Disturbance in Esports Athletes}

Based on the principles of traditional sleep interventions, guidelines can be adapted to fit the lifestyle of esports athletes. Compared to the traditional approach for sleep interventions, which usually consist of psychoeducation and/or several week- ly sessions, a flexible approach is necessary to accommodate training schedules and important matches.

\section{Managing game genre-related effects}

Issues that come up in treatment may vary depending on whether the game genre is team-or individual-player based. MOBA games such as League of Legends are team-based and training times (and sleeping times) usually occur similarly for all team members. Thus, group interventions may be necessary for team-based game genres as the team players cannot train alone. Additionally, game performance hinges on good teamwork strategies and communication between members, and sleep disturbance or sleep deprivation resulting in poor emotional regulation may be detrimental in working together as a team [52]. In contrast, individual player games such as FPS genres can benefit both from individual or group formats.

\section{Unconventional training times}

Esports athletes often train late at night. This may be due to training matches ("scrims") with teams that are in different time zones and also the delayed sleep tendencies of these young esports athletes. Designing interventions to work within these time parameters are imperative to a successful intervention with this population. Additionally, training times may not end until early morning (e.g., 1:00 am), and esports athletes may not retire to their sleep quarters until after sunrise. Thus, practicing sleep guidelines similar to shift workers such as incorporating eyeglasses that block blue light after training may help improve sleep disturbance [53].

\section{Cognitive therapy specific to esports athletes}

Many esports athletes may believe that sacrificing sleep to train excessively will enhance their performance, or sleeping less will make them perform better. Additionally, esports athletes may worry about the consequences of not getting enough sleep before an important match and put too much pressure on themselves trying to sleep. Psychoeducation based on the science of sleep highlighting sleep deprivation studies that have found reduced response speed (that can correspond to slower mouse-click speed) may be helpful to players in making the connection between sleep and game performance [17,54]. Additionally, traditional cognitive-behavioural therapy techniques such as socratic reasoning, cost-benefit analysis and behavioural experiments may be helpful in replacing dysfunctional and unhelpful thoughts about sleep. For example, asking esports athletes to perform a behavioural experiment where they extend their sleep duration and compare their game performance to days where they have sacrificed their sleep can help challenge their beliefs about sacrificing sleep.

One downside of traditional cognitive therapy is that there is a considerable amount of time investment required from esports athletes. Based on the adolescent sleep intervention litera- 
ture, one of the most common and accepted forms of cognitive therapy is mindfulness-based stress reduction (MBSR) [55-57]. MBSR introduces simple techniques such as the body scan that requires little time to teach and learn (1-2 $\mathrm{min})$ and implement (15 min per night). Thus, mindfulness-based techniques that have more convenient delivery may be an alternative to traditional cognitive restructuring that can be more conducive with the schedules of esports athletes.

\section{Importance of wind-down time}

Training and participating in game matches for esports athletes requires a high level of attention and concentration, and can cause stress that results in hyperarousal. Hyperarousal interferes with sleep and can cause insomnia [58,59], so incorporating a wind-down time to de-arouse in preparation for sleep is important for esports athletes. Traditional relaxation techniques such as progressive muscle relaxation, breathing retraining, meditation, and imagery rehearsal can be incorporated into treatment before bedtime, as well as including the mindfulness body scan technique.

\section{Adjusting to jet lag}

Being an esports athlete entails frequent traveling across multiple time zones [9], so sleep interventions should incorporate education on behavioural methods that can be implemented before, during and after travel to mitigate the effects of jet lag (i.e. circadian misalignment) and travel fatigue. To summarise, current recommendations [60] for traditional athletes suggest a combination of appropriately timed light exposure and exogenous melatonin that accounts for the timing of an individual's circadian rhythm and the direction of travel (eastward vs. westward travel). There may be some benefit to phase shifting prior to departure and during travel, but this needs to be managed carefully to prevent unintended consequences (e.g., sleep loss).

\section{Motivational strategies}

Motivation is critical for behavioural change yet it has been neglected as a treatment component in sleep interventions for traditional athletes $[10,61]$. In esports, motivation to make sleeprelated behavioural change may be a particularly important consideration because the majority of esports athletes are young, and young people are typically apathetic (and sometimes resistant) to this type of change $[62,63]$. A recent study by Micic et al. [64] that evaluated the readiness to change model [65] for adolescent sleep behaviour found that 'desire' (i.e., how much an individual wants/wishes to change), 'ability' (i.e., self-perception of capability to create change), and 'commitment' (i.e., intention or obligation to change) were the best predictive motivational components of sleep treatment compliance. Hence, incorporating motivational content may enhance sleep and performance outcomes.

\section{Early intervention}

As noted in the Gaming Culture section, gaming culture may shape dysfunctional sleep-related beliefs from a young age. Hence, early intervention in player development via sleep health education may be one way in which to combat the influence of gaming culture, by fostering more helpful sleep related beliefs and behaviour. Anecdotally, the University of Queensland in Australia has developed a program for high school students aspiring to become professional esports athletes. In their program they included material that aimed to 1) highlight the consequences of insufficient sleep, 2) encourage better sleep habits, and 3) promote a healthy balance between esports and sleep. Although this program has not been formally evaluated, there is some evidence that suggests early sleep intervention for children and adolescents can produce sleep benefits [66,67]. Additionally, evidence suggests that parental regulation of bedtime [68] and media [69] can help to protect adolescent sleep; therefore including parents in early intervention programs may also have added utility.

\section{CONCLUSION}

The function of sleep in esports has received minimal attention, yet research from our group and others is starting to provide critical insight on this matter. We have previously identified universal risk factors for sub-optimal sleep in esports such as caffeine, travel and pre-competition anxiety. Additionally, in the current review we propose that gaming culture and game genre may also play a role in negatively influencing sleep, via cognitive and behavioural pathways. Conceptually, adapting Spielman's 3-P model to esports is one way in which to formulate how these risk factors contribute to the development and maintenance of sub-optimal sleep. Moreover, from an intervention perspective, we propose that contemporary athlete sleep interventions can be suitably modified for use in esports but must comprehensively take into account all 3-P factors (i.e., predisposing, precipitating, perpetuating). Importantly, an esports sleep intervention needs to be flexible, address the practical and developmental needs of esports athletes, and include key team decision makers such as coaches and managers.

\section{Acknowledgments}

None.

\section{Conflicts of Interest}

The authors have no financial conflicts of interest.

\section{Authors' Contribution}

All authors were involved in the formulation of the study concept and design. Daniel Bonnar, Sangha Lee and Sooyeon Suh jointly completed the initial draft of the manuscript. Michael Gradisar and Sooyeon Suh edited multiple revisions of the manuscript. 


\section{REFERENCES}

1. Witkowski E. On the digital playing field: how we "do sport" with networked computer games. Games Cult 2012;7:349-74.

2. Newzoo. 2018 Global Esports market report. Amsterdam: Newzoo; 2018.

3. Wimbledon. Prize money and finance. [cited 2016 Dec 12]. Available from: https://www.wimbledon.com/en_GB/about_wimbledon/prize_ money_and_finance.html.

4. The Fortnite Team. The Fortnite World Cup: a record setting tournament. Epic games [online] 2019 Aug 1 [cited 2019 Nov 8]. Available from: https://www.epicgames.com/fortnite/competitive/en-US/news/ the-fortnite-world-cup-a-record-setting-tournament.

5. Hayward A. Olympics - backed $\$ 500 \mathrm{k}$ Intel World Open ties into 2020 Tokyo games with rocket league, street fighter V. The Esports Observer [online] 2019 Sep 11 [cited 2019 Nov 8]. Available from: https://esportsobserver.com/intel-world-open-2020/.

6. Himmelstein D, Liu Y, Shapiro JL. An exploration of mental skills among competitive League of Legend players. Int J Gaming Comput Mediat Simul 2017;9:1-21.

7. Goel N. Neurobehavioral effects and biomarkers of sleep loss in healthy adults. Curr Neurol Neurosci Rep 2017;17:89.

8. Lowe CJ, Safati A, Hall PA. The neurocognitive consequences of sleep restriction: a meta-analytic review. Neurosci Biobehav Rev 2017;80:586604.

9. Bonnar D, Castine B, Kakoschke N, Sharp G. Sleep and performance in Eathletes: for the win! Sleep Health 2019;5:647-50.

10. Bonnar D, Bartel K, Kakoschke N, Lang C. Sleep interventions designed to improve athletic performance and recovery: a systematic review of current approaches. Sports Med 2018;48:683-703.

11. merriam-webster.com [homepage on the Internet]. Springfield, MA: Merriam-Webster's collegiate dictionary [cited 2019 Nov 10]. Available from: https://www.merriam-webster.com/dictionary/culture.

12. Arslan S, Kocoglu D, Durmus M. Cultural beliefs affecting sleep duration. Sleep Biol Rhythms 2015;13:287-96.

13. Burgess DJ, Naughton GA. Talent development in adolescent team sports: a review. Int J Sports Physiol Perform 2010;5:103-16.

14. Samuels CH, Alexander BN. Sleep, recovery and human performance. Alberta: Canadian Sport Institute;2019.

15. Taylor TL. Raising the stakes: E-sports and the professionalization of computer gaming. Cambridge, MA: The MIT Press;2012.

16. Lewis JM, Trinh P, Kirsh D. A corpus analysis of strategy video game play in starcraft: brood war. CogSci 2011;33:687-92.

17. Van Dongen HP, Maislin G, Mullington JM, Dinges DF. The cumulative cost of additional wakefulness: dose-response effects on neurobehavioral functions and sleep physiology from chronic sleep restriction and total sleep deprivation. Sleep 2003;26:117-26.

18. Johnson MR, Woodcock J. 'It's like the gold rush': the lives and careers of professional video game streamers on Twitch.tv. Inf Commun Soc 2019; 22:336-51.

19. twitchtracker.com [homepage on the Internet]. San Francisco, CA: Twitch Tracker [cited 2019 Nov 10]. Available from: https://twitchtracker.com/ninja.

20. Goodling L. Streamer sets the record for most hours streamed in a single month on Twitch. Dot Esports [online] 2019 Apr 7 [cited 2019 Nov 10]. Available from: https://dotesports.com/streaming/news/streamer-sets-the-record-for-most-hours-streamed-in-a-single-month-ontwitch.

21. Lelchook A, Sully de Luque M. Cross-cultural training in industrial and organizational psychology. In: Wright JD. International encyclopedia of the social \& behavioral science. 2nd ed. Amsterdam: Elsevier;2015;334-41.

22. Zagała K, Strzelecki A. eSports evolution in football game series. Phys Cult Sport Stud Res 2019;83:50-62.

23. Deleuze J, Christiaens M, Nuyens F, Billieux J. Shoot at first sight! First person shooter players display reduced reaction time and compromised inhibitory control in comparison to other video game players. Comput Hum Behav 2017;72:570-6.

24. Mora-Cantallops M, Sicilia MA. MOBA games: a literature review. Entertain Comput 2018;26:128-38.

25. Baltezarević R, Baltezarević B. The impact of video games on the esports formation. Facta Universitatis, Series: Phys Edu Sport 2018;16:137-47.

26. Nedelec M, Aloulou A, Duforez F, Meyer T, Dupont G. The variability of sleep among elite athletes. Sports Med Open 2018;4:34.

27. Lastella M, Roach GD, Halson SL, Sargent C. Sleep/wake behaviours of elite athletes from individual and team sports. Eur J Sport Sci 2015;15: 94-100.

28. Dunican IC, Eastwood PR. Sleep is an important factor when considering rugby union player load. Br J Sports Med 2017;51:1640.

29. Schaal K, Tafflet M, Nassif H, Thibault V, Pichard C, Alcotte M, et al. Psychological balance in high level athletes: gender-based differences and sport-specific patterns. PLoS One 2011;6:e19007.

30. Emsellem HA, Murtagh KE. Sleep apnea and sports performance. Clin Sports Med 2005;24:329-41.

31. Tremblay MS, Willms JD. Is the Canadian childhood obesity epidemic related to physical inactivity? Int J Obes Relat Metab Disord 2003;27: $1100-5$.

32. Stettler N, Signer TM, Suter PM. Electronic games and environmental factors associated with childhood obesity in Switzerland. Obes Res 2004; 12:896-903.

33. Ballard M, Gray M, Reilly J, Noggle M. Correlates of video game screen time among males: body mass, physical activity, and other media use. Eat Behav 2009;10:161-7.

34. Dunton GF, Berrigan D, Ballard-Barbash R, Graubard B, Atienza AA. Joint associations of physical activity and sedentary behaviors with body mass index: results from a time use survey of US adults. Int J Obes (Lond) 2009;33:1427-36.

35. Suppiah HT, Low CY, Chia M. Effects of sports training on sleep characteristics of Asian adolescent athletes. Biol Rhythm Res 2015;46:523-36.

36. Wong K. StarCraft 2 and the quest for the highest APM. Engadget [online] 2014 Oct 24 [cited 2019 Nov 10]. Available from: https://www. engadget.com/2014/10/24/starcraft-2-and-the-quest-for-the-highestapm/.

37. Qaseem A, Kansagara D, Forciea MA, Cooke M, Denberg TD. Management of chronic insomnia cisorder in adults: a clinical practice guideline from the American College of Physicians. Ann Intern Med 2016;165: 125-33.

38. Siebern AT, Suh S, Nowakowski S. Non-pharmacological treatment of insomnia. Neurotherapeutics 2012;9:717-27.

39. Spielman AJ, Caruso LS, Glovinsky PB. A behavioral perspective on insomnia treatment. Psychiatr Clin North Am 1987;10:541-53.

40. Carskadon MA. Patterns of sleep and sleepiness in adolescents. Pediatrician 1990;17:5-12.

41. Roenneberg T, Kuehnle T, Pramstaller PP, Ricken J, Havel M, Guth A, et al. A marker for the end of adolescence. Curr Biol 2004;14:R1038-9.

42. Robinson JA, Alexander DJ, Gradisar MS. Preparing for Year 12 examinations: predictors of psychological distress and sleep. Aust J Psychol 2009;61:59-68.

43. Roberts SSH, Teo WP, Warmington SA. Effects of training and competition on the sleep of elite athletes: a systematic review and meta-analysis. Br J Sports Med 2019;53:513-22.

44. Crowley SJ, Acebo C, Carskadon MA. Sleep, circadian rhythms, and delayed phase in adolescence. Sleep Med 2007;8:602-12.

45. Mah CD, Mah KE, Kezirian EJ, Dement WC. The effects of sleep extension on the athletic performance of collegiate basketball players. Sleep 2011;34:943-50.

46. Schwartz J, Simon RD Jr. Sleep extension improves serving accuracy: a study with college varsity tennis players. Physiol Behav 2015;151:541-4.

47. Irish LA, Kline CE, Gunn HE, Buysse DJ, Hall MH. The role of sleep hygiene in promoting public health: a review of empirical evidence. Sleep Med Rev 2015;22:23-36. 
48. Schaal K, LE Meur Y, Louis J, Filliard JR, Hellard P, Casazza G, et al. Whole-body cryostimulation limits overreaching in elite synchronized swimmers. Med Sci Sports Exerc 2015;47:1416-25.

49. Zhao J, Tian Y, Nie J, Xu J, Liu D. Red light and the sleep quality and endurance performance of Chinese female basketball players. J Athl Train 2012;47:673-8.

50. Miles KH, Clark B, Fowler PM, Miller J, Pumpa KL. Sleep practices implemented by team sport coaches and sports science support staff: a potential avenue to improve athlete sleep? J Sci Med Sport 2019;22:74852.

51. Farahnak LR, Ehrhart MG, Torres EM, Aarons GA. The Influence of transformational leadership and leader attitudes on subordinate attitudes and implementation success. J Leadersh Organ Stud 2019;127:98111.

52. Talbot LS, McGlinchey EL, Kaplan KA, Dahl RE, Harvey AG. Sleep deprivation in adolescents and adults: changes in affect. Emotion 2010;10: 831-41.

53. Sasseville A, Benhaberou-Brun D, Fontaine C, Charon MC, Hebert M. Wearing blue-blockers in the morning could improve sleep of workers on a permanent night schedule: a pilot study. Chronobiol Int 2009;26: 913-25.

54. Dinges DF, Pack F, Williams K, Gillen KA, Powell JW, Ott GE, et al. Cumulative sleepiness, mood disturbance, and psychomotor vigilance performance decrements during a week of sleep restricted to $4-5$ hours per night. Sleep 1997;20:267-77.

55. Bootzin RR, Stevens SJ. Adolescents, substance abuse, and the treatment of insomnia and daytime sleepiness. Clin Psychol Rev 2005;25:629-44.

56. Blake M, Schwartz O, Waloszek JM, Raniti M, Simmons JG, Murray G, et al. The SENSE study: treatment mechanisms of a cognitive behavioral and mindfulness-based group sleep improvement intervention for at-risk adolescents. Sleep 2017;40.

57. Bartel K, Huang C, Maddock B, Williamson P, Gradisar M. Brief schoolbased interventions to assist adolescents' sleep-onset latency: comparing mindfulness and constructive worry versus controls. J Sleep Res 2018; 27:e12668.
58. Bonnet MH, Arand DL. Hyperarousal and insomnia: state of the science. Sleep Med Rev 2010;14:9-15.

59. Riemann D, Spiegelhalder K, Feige B, Voderholzer U, Berger M, Perlis $\mathrm{M}$, et al. The hyperarousal model of insomnia: a review of the concept and its evidence. Sleep Med Rev 2010;14:19-31.

60. Roach GD, Sargent C. Interventions to minimize jet lag after westward and eastward flight. Front Physiol 2019;10:927.

61. Halson SL, Lastella M. Amazing athletes with ordinary habits: why is changing behavior so difficult? Int J Sports Physiol Perform 2017;12: 1273-1274.

62. Moseley L, Gradisar M. Evaluation of a school-based intervention for adolescent sleep problems. Sleep 2009;3:334-341.

63. Cain N, Gradisar M, Moseley L. A motivational school-based intervention for adolescent sleep problems. Sleep Med 2011;12:246-251.

64. Micic G, Richardson C, Cain N, Reynolds C, Bartel K, Maddock B, et al. Readiness to change and commitment as predictors of therapy compliance in adolescents with delayed sleep-wake phase cisorder. Sleep Med 2019;55:48-55.

65. Amrhein PC, Miller WR, Yahne CE, Palmer M, Fulcher L. Client commitment language during motivational interviewing predicts drug use outcomes. J Consult Clin Psychol 2003;71:862-878.

66. Bonnar D, Gradisar M, Moseley L, Coughlin AM, Cain N, Short MA. Evaluation of novel school-based interventions for adolescent sleep problems: does parental involvement and bright light improve outcomes? Sleep Health 2015;1:66-74.

67. Gruber R, Somerville G, Bergmame L, Fontil L, Paquin S. School-based sleep education program improves sleep and academic performance of school-age children. Sleep Med 2016;21:93-100.

68. Short MA, Gradisar M, Wright H, Lack LC, Dohnt H, Carskadon MA. Time for bed: parent-set bedtimes associated with improved sleep and daytime functioning in adolescents. Sleep 2011;34:797-800.

69. Smith LJ, Gradisar M, King DL, Short M. Intrinsic and extrinsic predictors of video-gaming behaviour and adolescent bedtimes: the relationship between flow states, self-perceived risk-taking, device accessibility, parental regulation of media and bedtime. Sleep Med 2017;30:64-70. 